\title{
Occurrence of Pulmonary Adenomatosis (Jaagsiekte) in sheep of animal research institute: the strategy of working with pulmonary adenomatosis in a flock of sheep
}

F Amini, A Mahdavi

Animal Science Reaserches of Iran, Karaj, Tehran, Islamic Republic of Iran

Email:fred_amini@yahoo.com

Introduction OPA is a contagious, viral, neoplastic disease of the lungs of sheep (Palmarini et al, 1997)). It is a contagious tumour of sheep, a progressive respiratory disease, principally affecting adult animals. Two viruses, a herpes virus and a retrovirus (slow virus), have been associated with the disease, but only the latter has an aetiological role (Palmarini et al, 1999). Natural transmission seems to occur generally by the respiratory route. Close contact (e.g., at feeding troughs) may spread the virus (Palmarini et al, 1997). The tumours produce clinical signs when they become sufficiently large, or numerous enough to interfere with respiration. Affected sheep lose weight and show respiratory symptoms. Moist rales may be heard even without a stethoscope. Forced lowering of the head often causes frothy mucus to run from the nostrils. There is no specific treatment or vaccine available. The best is once the diagnosis confirmed and animals showing signs suggestive of pulmonary adenomatosis should be removed from the flock. A diagnostic test for the disease is by raising the hindquarters and lowering the head of affected sheep may cause frothy mucoid fluid to run from the nostrils The objective of the present study was to monitor the cases of OPA in a sheep flock that consisted of 5 breeds and identify how long it will take to eradicate the disease from the flock.

Material and method Every month the adult sheep were tested to find the suspected cases (for numbers of sheep see Table 1). Those which suffer from pulmonary distress such as difficult breathing, tachypenia, abdominal breathings, emaciated were examined thoroughly. The rear of affected sheep were lifted if excess fluid run from the nose, then it was diagnosed as an OPA case and slaughtered. In carcass inspection the lungs were examined for the lesions of disease and then sent to pathology lab for confirmation of the diagnosis. In post-mortem the lungs were enlarged (as much as triple their normal size) grey-yellow non collapsible (do not collapse when the thoracic cavity is opened) with rubbery consistency. In cross of lung parenchyma showing a meaty appearance mostly in ventral part of the lung. The mediastinal lymph nodes were enlarged as like a small tube. The lungs sink in water when submerged. The disease came under control after 4 years in this flock. In $5^{\text {th }}$ year of this study no more cases was seen. The design was completely randomized with imbalanced replication and mean comparison was performed by Duncan's multiple comparison test.

Results As the data in Table 1 shows the merinos and its crosses have lowest susceptibility among imported and native breeds of sheep. Suffolk and kiosi breed with their crosses had highest susceptibility to the disease. These two breeds are high producer of meat and milk accordingly. There are not any significant differences between the susceptibility of native breeds.

Table 1 Breeds, total no of sheep, no of sheep affected, and \% of sheep affected

\begin{tabular}{llll}
\hline \hline Breed of sheep & $\begin{array}{l}\text { Total number of sheep } \\
\text { examined in 5 years of study }\end{array}$ & Number affected during 5 year period & \% of sheep affected \\
\hline merinos and crosses & 231 & 33 & $14.3^{\mathrm{b}}$ \\
Kiosi and crosses & 125 & 32 & $25.6^{\mathrm{a}}$ \\
Suffolk and crosses & 195 & 47 & $24.1^{\mathrm{a}}$ \\
Mogani breed & 33 & 7 & $21.2^{\mathrm{ab}}$ \\
Shal breed & 66 & 13 & $19.6^{\mathrm{ab}}$ \\
Total & 650 & 132 & 20.3 \\
\hline \hline
\end{tabular}

Values with different superscripts denote significance within column
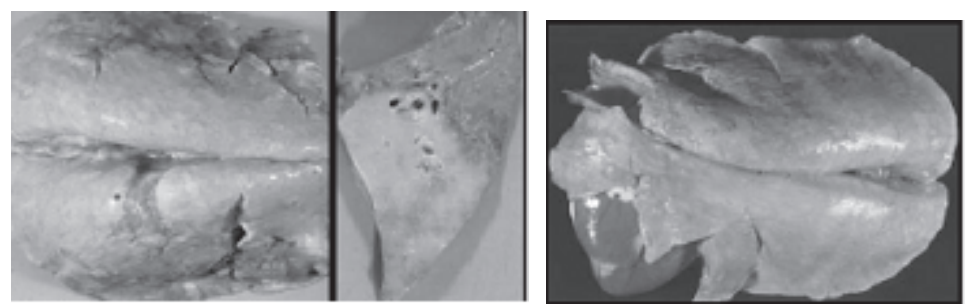

\section{Conclusion}

By selection of clinically positive cases and their slaughter, the disease appeared to be controlled after 4 years of the regime, whereas it disappeared in 5 years. Early diagnosis and slaughter at the early stages of the illness enables the carcass to be used for human consumption. If the disease is not diagnosed until the later stages, the sheep emaciate and the carcass should be condemned, which causes heavy losses to farmers. The positive relationship between high productivity of sheep (meat and milk production) and occurrence of this disease in this trial needs further investigation.

\section{References}

Palmarini M., et al (1997). Trends in Microbiology 5, 478-483

Palmarini M., et al (1999). Journal of Virology 73, 6964-6972. 